\title{
Usage Examples
}

\section{Typescript/Mol*}

This requires node.js (https://nodejs.org/) to be installed. In a terminal, issue 'npm install -g molstar` to install the molstar package.

Then, use cif2bcif file.cif file.bcif to convert any cif file to a bcif file. See cif2bcif $-h$ for more options. To generate code from cif dictionaries see help of the cifschema $-h$ command. For instance cifschema --preset mmCIF to generate TypeScript types describing mmCIF files for use with Mol*.

Java

This requires a Java Runtime Environment (https://www.java.com/en/download/) to be installed and the ciftools-java dependency to be imported e.g. using Maven:

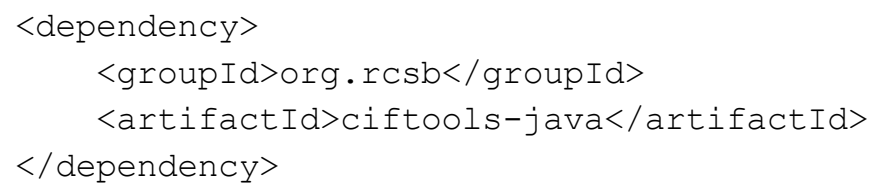

Then, any PDB identifier can be downloaded in cif format from RCSB PDB and the corresponding bcif file will be written to the specified path.

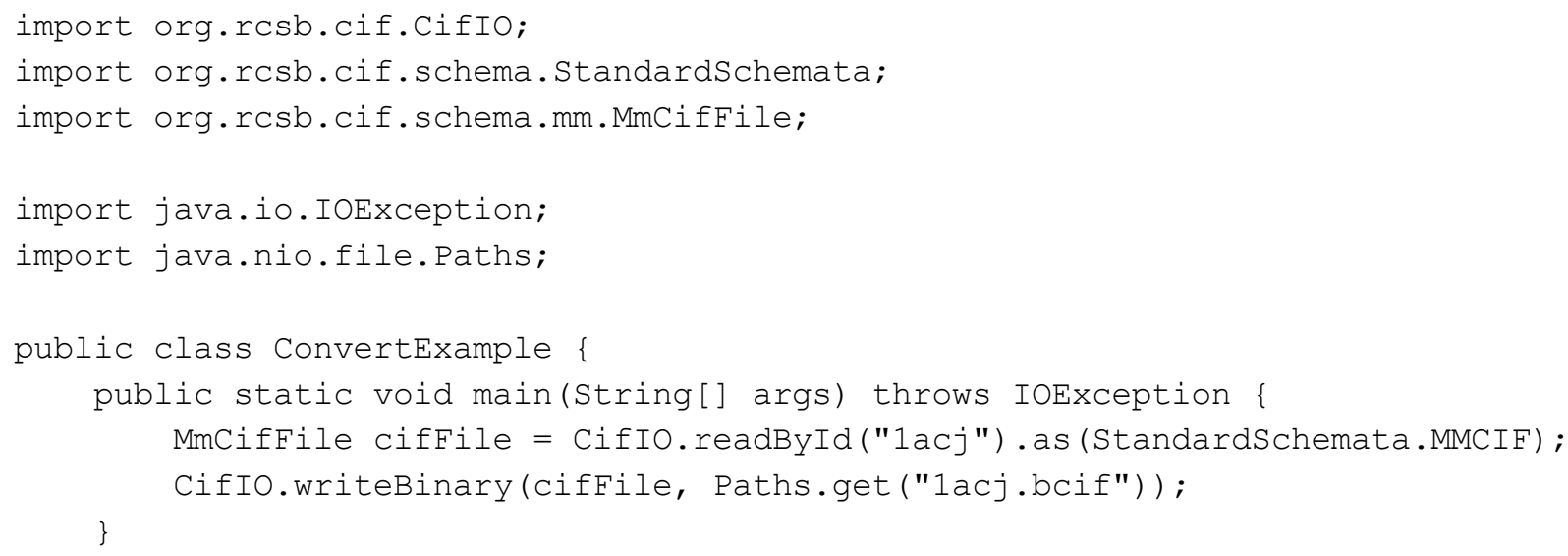

